\title{
Evaluación de niveles, situaciones generadoras y manifestaciones de estrés académico en alumnos de tercer y cuarto año de una Facultad de Estomatología
}

Bedoya SA, Perea M, Ormeño R.Evaluación de niveles, situaciones generadoras y manifestaciones de estrés académico en alumnos de tercer y cuarto año de una Facultad de Estomatología. Rev Estomatol Herediana 2006; 16(1): 15 - 20.

\section{Silvana Aranceli Bedoya Lau ${ }^{1}$ Miguel Perea $\mathrm{Paz}^{2}$ Rosario Ormeño Martínez ${ }^{3}$}

Cirujano-Dentista.

2Docente de Departamento Académico de Estomatología del Niño y del Adolescente. Facultad de Estomatología.

${ }^{3}$ Docente del Departamento de Ciencias y Filosofia de la Escuela de Tecnología Médica. Facultad de Medicina Humana.

Universidad Peruana Cayetano Heredia.

\section{Correspondencia}

Silvana Aranceli Bedoya Lau Av. Venezuela 625-206 - Lima 05, Perú Teléfono: 99456051

e-mail: silvanablau@ hotmail.com

\section{Aceptado para publicación :}

23 de octubre del 2006.

\section{RESUMEN}

El presente estudio tuvo como propósito evaluar los niveles, situaciones generadoras y manifestaciones físicas, psicológicas y comportamentales en 118 alumnos del tercer y cuarto año de la Facultad de Estomatología de la Universidad Peruana Cayetano Heredia. La distribución de la

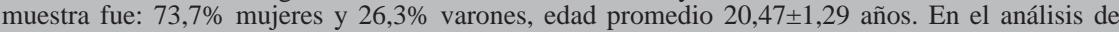
los resultados se utilizaron los porcentajes para determinar la presencia de estrés, la comparación directa de medias para describir la intensidad con que se presentaron las situaciones generadoras y manifestaciones de estrés y el análisis correlacional se realizó a través del coeficiente Tau de Kendall. Los resultados revelan que el 96,6\% de alumnos reconocieron la presencia de estrés académico, siendo los alumnos de tercer año los que presentaron los niveles más altos, las mujeres presentaron niveles más bajos de estrés en relación a los varones. Las situaciones mayormente generadoras de estrés fueron tiempo para cumplir con las actividades, sobrecarga de tareas, trabajos de cursos y responsabilidad por cumplir obligaciones académicas. Las manifestaciones físicas, psicológicas y comportamentales se presentaron con mayor intensidad en los varones; siendo las psicológicas, las que tuvieron mayor presencia e intensidad en ambos sexos y años de estudios. Finalmente se encontró que el nivel de estrés académico se relacionó positivamente con todas las situaciones generadoras y manifestaciones físicas y psicológicas; así como se relacionó negativamente con algunos comportamientos indagados.

Palabras claves: ESTUDIANTES DE ODONTOLOGÍA / ESTRÉS / AUTOPERCEPCIÓN.

Assessment of levels, generating situations and manifestations of academic stress in dental students of the third and fourth year of the career.

ABSTRACT

The objective of this study was to assess levels, generating situations and physical, psychological and behavioral manifestations of academic stress in 118 students belonging to the third and fourth year of the Faculty of Stomatology at Cayetano Heredia University. The sample was formed by $73.7 \%$ females and $26.3 \%$ males, with a mean age of $20.47 \pm 1.29$ years. Percentages were used to analyses stress presence, direct comparison of medias was used for the intensity with which generating situations and stress manifestations occurred. The correlation analysis was carried out by Tau coefficient of Kendall. The results revealed that $96.6 \%$ of the students recognized the presence of academic stress; third year students presented the highest levels, women manifested lower levels of stress in comparison with men. Main generations situations of stress were lack of time to accomplish clinical activities, homework overlode, and responsability to accomplish academic demands. Physical, psychologic and behavior manifestations occurred with more intensity in men, being the psychologic manifestations the highest presence and intensity in both genders. Finally, it was found that the levels of academic stress were positively related with all the generating situations, and physical and psychologic manifestations; as well as it was negatively related with some investigated conducts.

Keywords: DENTAL STUDENTS / STRESS / SELF CONCEPT.

\section{Introducción}

El estrés se puede definir como el mecanismo general del organismo para adaptarse a todas las influencias, cambios, exigencias y tensiones a los que podía estar expuesto la persona, siendo común en todos estos procesos la forma como el cuerpo trata de adaptarse (1).

La cantidad de estrés produce un estado de alerta necesario para obtener un rendimiento físico y mental que permita ser productivos y creativos. El estrés da lugar a una sensación de confianza, de control y de capacidad para abordar y llevar a buen termino tareas, retos y demandas concretas (2).

Hernández, Polo y Poza en 1994 (3), definieron el estrés académico como aquel que se produce en el ámbito educativo por ende, podría afectar tanto a profesores en el abordaje de sus tareas docentes, como a estudiantes en cualquier nivel educativo, en especial el del ámbito universitario.

Los universitarios están sometidos a un conjunto de situaciones altamente estresantes debido a que suelen experimentar una falta de control sobre el nuevo ambiente, potencialmente generador de estrés (4).

Así, la exposición continua a estas situaciones estresantes pueden deteriorar en forma progresiva el funcionamiento del organismo con la consecuente aparición de problemas de salud (5-8). En este sentido, existen estudios donde se destacan varias fuentes de estrés en la educación odontológica y se analizan aspectos relacionados al estrés académico (9-14).

El objetivo del presente estudio fue determinar la frecuencia, nivel, situaciones generadoras y manifestaciones del estrés académico auto-percibido por los alumnos del tercer y cuarto año de la Facultad de Estomatología de la Universidad Peruana Cayetano Heredia. 


\section{Materiales y método}

La población estuvo compuesta por 118 alumnos del tercer y cuarto año de la Facultad de Estomatología de la Universidad Peruana Cayetano Heredia, que aceptaron participar en el estudio firmando un consentimiento informado.

Mediante un cuestionario desarrollado en forma colectiva y dentro del aula donde recibían clases teórico-practicas se evaluaron las siguientes variables :

- Situaciones generadoras de estrés (estresores). Se refiere a los eventos ocurridos dentro del ámbito universitario que generan potencialmente estrés académico en los estudiantes. Se evaluaron cinco estresores: (1)Competencia con compañeros. (2)Responsabilidad por labores académicas. (3)Sobrecarga de tareas y trabajos de cursos. (4)Evaluación de los profesores en el área clínica. (5)Tiempo para cumplir con las actividades.

- Reacciones o síntomas físicos ante el estrés. Se refiere a la aparición de respuestas físicas asociadas al estrés. Se evaluaron cinco reacciones físicas: (1)Trastornos en el sueño. (2)Fatiga crónica (cansancio permanente). (3)Dolores de cabeza o migrañas. (4)Problemas de digestión, dolor abdominal o diarrea. (5)Somnolencia o mayor necesidad de dormir.

- Reacciones o síntomas psicológicos ante el estrés. Se refiere a la aparición de respuestas psicológicas asociadas al estrés. Se evaluaron cinco reacciones psicológicas: (1)Incapacidad de relajarse y estar tranquilo. (2)Sentimientos de depresión y tristeza. (3)Ansiedad, mayor predisposición a temores. (4)Problemas de concentración. (5)Sensación de tener la mente vacía.

- Reacciones o síntomas comportamentales ante el estrés. Se refiere a la frecuencia de comportamientos asociados al estrés. Se evaluaron cinco comportamientos: (1)Fumar. (2)Conflictos o tendencia a discutir. (3)Aislamiento de los demás. (4)Desgano para realizar obligaciones académicas. (5)Ausentismo de las clases.

- Percepción subjetiva del estrés. Se refiere a la percepción del individuo frente a cada una de las situaciones planteadas.

Para determinar la presencia de estrés académico se emplearon porcentajes, mientras que para describir el nivel de estrés académico auto-percibido y la intensidad con que se presentaron las situaciones generadoras, reacciones físi- cas, reacciones psicológicas y comportamientos ante el estrés se emplearon la media aritmética y desviación estándar.

Las situaciones generadoras, reacciones físicas, psicológicas y comportamientos ante el estrés fueron comparadas según sexo y año de estudios empleando la comparación directa de medias. Se utilizó el coeficiente de correlación Tau de Kendall para evaluar la relación entre el nivel de estrés auto-percibido y las situaciones generadoras, reacciones físicas, psicológicas y comportamientos ante el estrés. Finalmente, la fuerza de la asociación fue interpretada de acuerdo a la escala propuesta por Colton.

\section{Resultados}

De los 118 alumnos encuestados el $96,6 \%$ reconocieron la presencia de estrés académico (Fig. 1).

En relación al nivel de estrés académico auto-percibido los alumnos del tercer año presentaron un nivel de estrés

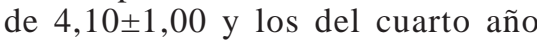
$3,86 \pm 1,04$. Las mujeres presentaron un nivel de estrés de 3,96 $\pm 0,98$ y los varones 4,06 $\pm 1,12$

En relación con los estresores en estudiantes de tercer año se obtuvieron mayores puntajes para varones en todos los estresores, sin embargo, en estudiantes de cuarto año las mujeres obtuvieron mayores puntajes que los varones para todos los estresores a excepción de sobrecarga de tareas y trabajos de cursos. De los cinco estresores evaluados, el tiempo para cumplir con las actividades académicas fue al que los estudiantes de tercer y cuarto año asignaron un mayor puntaje (Tabla 1).

En relación con las reacciones físicas se encontró en los estudiantes de tercer año puntajes mayores para varones que para mujeres en todas las reacciones físicas a excepción de dolores de cabeza o migrañas; sin embargo, en estudiantes de cuarto año los varones obtuvieron mayores puntajes que las mujeres para fatiga crónica y dolores de cabeza o migrañas. La somnolencia o mayor necesidad de dormir obtuvo similares puntajes en varones y mujeres del cuarto año. De las cinco reacciones físicas evaluadas, la somnolencia o mayor necesidad de dormir fue la reacción de mayor putaje en los estudiantes de tercer y cuarto año (Tabla 2).

En relación con las reacciones psicológicas, se encontró en estudiantes de tercer año los mayores puntajes en varones en todas las reacciones psicológicas, igualmente, en estudiantes de cuarto año los varones obtuvieron los mayores puntajes para todas las reacciones psicológicas a excepción de la incapacidad para relajarse y estar tranquilo. De las cinco reacciones psicológicas evaluadas, la incapacidad para relajarse y estar tranquilo obtuvo el mayor puntaje en los estudiantes de tercer y cuarto año asignaron un mayor puntaje (Tabla 3).

En relación con los comportamientos ante el estrés, se encontró en estudiantes de tercer año los mayores puntajes en mujeres en todos los comportamientos a excepción del aislamiento de los demás. En estudiantes de cuarto año los varones obtuvieron mayores puntajes para todos los comportamientos a excepción de fumar. De los cinco comportamientos evaluados, el desgano para realizar labores académicas obtuvo el mayor puntaje en los estudiantes de tercer y cuarto año (Tabla 4).

La relación entre el nivel de estrés académico auto-percibido y los estresores, reacciones físicas, reacciones psicológicas y comportamientos ante el estrés, se obtuvo aplicando el coeficiente de correlación tau de Kendall (Tabla 5).

El nivel de estrés estuvo relacionado con todos los estresores evaluados, existiendo moderado grado de correlación con la responsabilidad por cumplir obligaciones académicas y cierto grado de correlación con los demás estresores. Se encontró una relación con todas las reacciones físicas, existiendo cierto grado de correlación con la fatiga crónica, trastornos del sueño y somnolencia o mayor necesidad de dormir y el rubro otros, así como también escasa correlación con las demás reacciones físicas (Tabla 6).

Por otra parte se encontró una relación con todas las reacciones psicológicas, existiendo una correlación con la incapacidad de relajarse y estar tranquilo y el rubro otros así como también una correlación con las demás reacciones psicológicas. Finalmente, se encontró una relación inversa con tres de los comportamientos evaluados, existiendo buen grado de correlación con el ausentismo de clases, moderado grado de correlación con el rubro otros, y escaso grado de correlación con fumar. La relación fue directa y de escaso grado de correlación con los otros tres comportamientos evaluados (Tabla 6). 


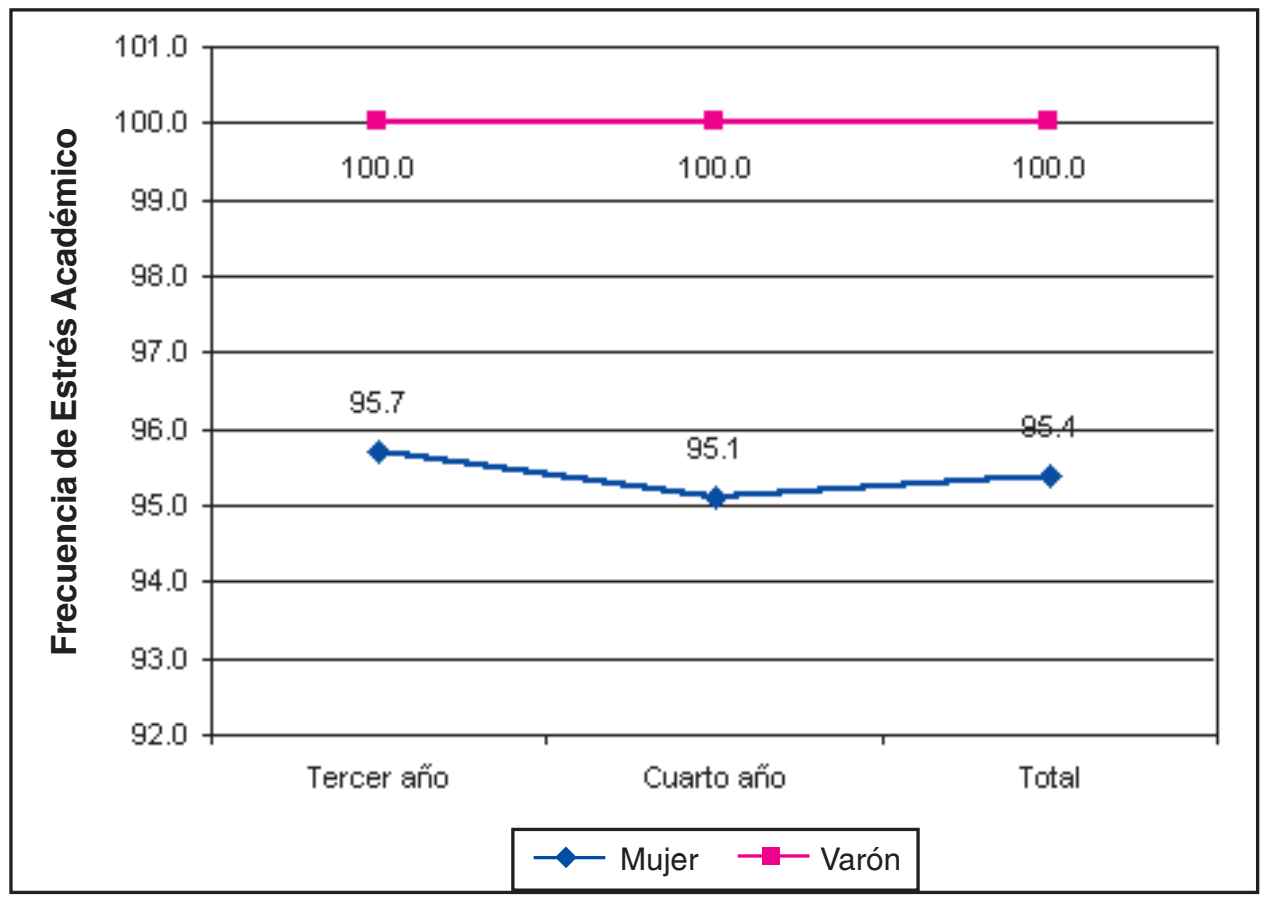

Fig. 1. Frecuencia de estrés académico según sexo y año de estudios en alumnos de la Facultad de Estomatología (2005-I)

Tabla 1. Puntaje de estresores según sexo y año de estudios de los alumnos.

\begin{tabular}{|c|c|c|c|c|c|c|}
\hline estresores & $\begin{array}{l}\text { Mujer } \\
(n=44) \\
X(D E)\end{array}$ & $\begin{array}{l}\text { Tercer año } \\
\text { Varón } \\
(\mathrm{n}=19) \\
\mathrm{X}(\mathrm{DE})\end{array}$ & $\begin{array}{l}\text { Total } \\
(n=63) \\
X(D E)\end{array}$ & $\begin{array}{l}\text { Mujer } \\
(n=39) \\
X(D E)\end{array}$ & $\begin{array}{l}\text { Cuarto año } \\
\text { Varón } \\
(\mathrm{n}=12) \\
\mathrm{X}(\mathrm{DE})\end{array}$ & $\begin{array}{l}\text { Total } \\
(n=51) \\
X(D E)\end{array}$ \\
\hline Competencia & $2,70(1,05)$ & $3,11(0,81)$ & 2,83(0,99) & $2,67(1,03)$ & $2,58(1,17)$ & $2,65(1,06)$ \\
\hline $\begin{array}{l}\text { Responsabilidad por } \\
\text { labores académicas } \\
\text { Sobrecarga de tareas }\end{array}$ & $3,82(1,02)$ & 4,21(0,92) & $3,94(1,00)$ & $3,77(1,06)$ & $3,75(1,06)$ & $3,76(1,05)$ \\
\hline $\begin{array}{l}\text { y trabajos de cursos } \\
\text { Evaluación de los }\end{array}$ & $4,11(1,04)$ & $4,58(0,61)$ & $4,25(0,95)$ & $3,56(1,14)$ & $3,75(0,97)$ & $3,61(1,10)$ \\
\hline $\begin{array}{l}\text { profesores por módulo } \\
\text { Tiempo para cumplir }\end{array}$ & $3,45(1,13)$ & $4,16(0,90)$ & $3,67(1,11)$ & $3,28(1,00)$ & $2,83(1,03)$ & $3,18(1,01)$ \\
\hline con las actividades & $4,43(0,93)$ & $4,63(0,68)$ & $4,49(0,86)$ & $4,18(1,14)$ & $4,17(0,94)$ & $4,18(1,09)$ \\
\hline
\end{tabular}

Tabla 2. Puntaje de las reacciones físicas según sexo y año de estudios de los alumnos.

\begin{tabular}{|c|c|c|c|c|c|c|}
\hline Reacción física & $\begin{array}{l}\text { Mujer } \\
(n=44) \\
X(D E)\end{array}$ & $\begin{array}{l}\text { Tercer año } \\
\text { Varón } \\
(\mathrm{n}=19) \\
\mathrm{X}(\mathrm{DE})\end{array}$ & $\begin{array}{l}\text { Total } \\
(\mathrm{n}=63) \\
\mathrm{X}(\mathrm{DE})\end{array}$ & $\begin{array}{l}\text { Mujer } \\
(n=39) \\
\text { X(DE) }\end{array}$ & $\begin{array}{l}\text { Cuarto año } \\
\text { Varón } \\
(\mathrm{n}=12) \\
\mathrm{X}(\mathrm{DE})\end{array}$ & $\begin{array}{l}\text { Total } \\
(\mathrm{n}=51) \\
\mathrm{X}(\mathrm{DE})\end{array}$ \\
\hline Trastornos en el sueño & $2,84(1,31)$ & $3,26(1,52)$ & $2,97(1,38)$ & $2,95(1,28)$ & $2,33(1,16)$ & $2,80(1,27)$ \\
\hline $\begin{array}{l}\text { Fatiga crónica (cansancio } \\
\text { permanente) } \\
\text { Dolores de cabeza }\end{array}$ & $3,57(1,02)$ & $3,84(1,26)$ & $3,65(1,10)$ & $3,15(1,27)$ & $3,42(1,17)$ & $3,22(1,24)$ \\
\hline o migrañas & $2,89(1,21)$ & $2,84(1,34)$ & $2,87(1,24)$ & $2,74(1,35)$ & $3,00(1,13)$ & $2,80(1,30)$ \\
\hline $\begin{array}{l}\text { Problemas de digestión, } \\
\text { dolor abdominal o diarrea } \\
\text { Somnolencia o mayor }\end{array}$ & $2,25(1,26)$ & $2,68(1,53)$ & $2,38(1,35)$ & $2,67(1,31)$ & $2,50(1,31)$ & $2,63(1,30)$ \\
\hline necesidad de dormir & $3,93(1,04)$ & $3,95(1,51)$ & $3,94(1,19)$ & $3,67(1,24)$ & $3,67(1,30)$ & $3,67(1,24)$ \\
\hline
\end{tabular}


Tabla 3. Puntaje de las reacciones psicológicas según sexo y año de estudios en los alumnos.

\begin{tabular}{|c|c|c|c|c|c|c|}
\hline Reacción psicológica & $\begin{array}{l}\text { Mujer } \\
(n=44) \\
X(D E)\end{array}$ & $\begin{array}{l}\text { Tercer año } \\
\text { Varón } \\
(\mathrm{n}=19) \\
\mathrm{X}(\mathrm{DE})\end{array}$ & $\begin{array}{l}\text { Total } \\
(\mathrm{n}=63) \\
\mathrm{X}(\mathrm{DE})\end{array}$ & $\begin{array}{l}\text { Mujer } \\
(n=39) \\
X(D E)\end{array}$ & $\begin{array}{l}\text { Cuarto año } \\
\text { Varón } \\
(\mathrm{n}=12) \\
\mathrm{X}(\mathrm{DE})\end{array}$ & $\begin{array}{l}\text { Total } \\
(\mathrm{n}=51) \\
\mathrm{X}(\mathrm{DE})\end{array}$ \\
\hline $\begin{array}{l}\text { Incapacidad de relajarse } \\
\text { y estar tranquilo } \\
\text { Sentimientos de }\end{array}$ & $3,59(1,15)$ & $3,74(1,24)$ & $3,63(1,17)$ & $3,23(1,33)$ & $3,17(1,03)$ & $3,22(1,25)$ \\
\hline $\begin{array}{l}\text { depresión y tristeza } \\
\text { Ansiedad, mayor }\end{array}$ & $3,27(1,17)$ & $3,79(1,08)$ & $3,43(1,16)$ & $3,18(1,37)$ & $3,33(1,37)$ & $3,22(1,36)$ \\
\hline $\begin{array}{l}\text { predisposición a temores } \\
\text { Problemas de }\end{array}$ & $3,00(1,46)$ & $3,53(1,07)$ & $3,16(1,37)$ & $3,21(1,28)$ & $3,50(1,09)$ & $3,27(1,23)$ \\
\hline $\begin{array}{l}\text { concentración } \\
\text { Sensación de tener }\end{array}$ & $3,00(1,20)$ & $3,42(1,43)$ & $3,13(1,28)$ & $3,00(1,19)$ & $3,58(1,24)$ & $3,14(1,22)$ \\
\hline la mente vacía & $2,82(1,53)$ & $3,11(1,63)$ & $2,90(1,55)$ & $2,56(1,39)$ & $2,58(1,31)$ & $2,80(1,43)$ \\
\hline
\end{tabular}

Tabla 4. Puntaje de los comportamientos ante el estrés según sexo y año de estudios en los alumnos.

\begin{tabular}{|c|c|c|c|c|c|c|}
\hline Reacción psicológica & $\begin{array}{l}\text { Mujer } \\
(n=44) \\
X(D E)\end{array}$ & $\begin{array}{l}\text { Tercer año } \\
\text { Varón } \\
(\mathrm{n}=19) \\
\mathrm{X}(\mathrm{DE})\end{array}$ & $\begin{array}{l}\text { Total } \\
(n=63) \\
X(D E)\end{array}$ & $\begin{array}{l}\text { Mujer } \\
(n=39) \\
X(D E)\end{array}$ & $\begin{array}{l}\text { Cuarto año } \\
\text { Varón } \\
(\mathrm{n}=12) \\
\mathrm{X}(\mathrm{DE})\end{array}$ & $\begin{array}{l}\text { Total } \\
(n=51) \\
X(D E)\end{array}$ \\
\hline Fumar & $2,48(1,66)$ & $2,37(1,54)$ & $2,44(1,61)$ & $2,41(1,57)$ & $2,25(1,71)$ & $2,37(1,59)$ \\
\hline $\begin{array}{l}\text { Conflictos o tendencia } \\
\text { a discutir } \\
\text { Aislamiento de }\end{array}$ & $2,93(1,39)$ & $2,84(1,34)$ & $2,90(1,36)$ & $2,31(1,26)$ & $3,17(1,34)$ & $2,51(1,32)$ \\
\hline $\begin{array}{l}\text { los demás } \\
\text { Desgano para realizar }\end{array}$ & $2,36(1,38)$ & $2,37(1,21)$ & 2,37(1,32) & $2,13(1,06)$ & $2,33(1,56)$ & $2,18(1,18)$ \\
\hline $\begin{array}{l}\text { labores académicas } \\
\text { Ausentismo de }\end{array}$ & $3,00(1,38)$ & 2,95(1,39) & 2,98(1,37) & $2,77(1,20)$ & $3,17(1,27)$ & $2,86(1,22)$ \\
\hline las clases & $2,30(1,49)$ & $1,95(1,39)$ & $2,19(1,46)$ & $2,08(1,27)$ & $2,25(1,36)$ & $2,12(1,28)$ \\
\hline
\end{tabular}

Tabla 5. Relación entre nivel de estrés auto-percibido y cada evento evaluado en alumnos de la Facultad de Estomatología (2005-I)

\begin{tabular}{|c|c|c|}
\hline \multirow[t]{6}{*}{ Eventos Estresores } & & Tau \\
\hline & Responsabilidad por labores académicas & 0,56 \\
\hline & Sobrecarga de tareas y trabajos de cursos & 0,44 \\
\hline & Tiempo para cumplir con las actividades & 0,44 \\
\hline & Evaluación de los profesores en módulo & 0,35 \\
\hline & Competencia con compañeros & 0,29 \\
\hline Reaccion fisica & Fatiga crónica (cansancio permanente) & 0.42 \\
\hline & Trastornos en el sueño & 0,35 \\
\hline & Somnolencia o mayor necesidad de dormir & 0,32 \\
\hline & Dolores de cabeza o migrañas & 0,22 \\
\hline & Problemas de digestión, dolor abdominal o diarrea & 0,19 \\
\hline \multirow[t]{6}{*}{ Reacción psicológica } & & \\
\hline & Incapacidad de relajarse y estar tranquilo & 0,57 \\
\hline & Sentimientos de depresión y tristeza & 0,34 \\
\hline & Sensación de tener la mente vacía & 0,32 \\
\hline & Problemas de concentración0,30 & \\
\hline & Ansiedad, mayor predisposición a temores & 0,29 \\
\hline \multirow[t]{6}{*}{ Comportamientos } & & \\
\hline & Conflictos o tendencia a discutir & 0,23 \\
\hline & Desgano para realizar labores académicas & 0,16 \\
\hline & Aislamiento de los demás & 0,14 \\
\hline & Fumar & $-0,17$ \\
\hline & Absentismo de las clases & $-0,70$ \\
\hline
\end{tabular}




\section{Discusión}

En este estudio la presencia del estrés académico fue de 96,6\%, dato que difiere de los de Polo, Hernández y Poza (7) que reportan 100\% del estrés académico en universitarios y de Barraza $(15,16)$ el 2003 y 2005 donde los alumnos del postgrado reconocieron un $100 \%$ de estrés académico y los alumnos de educación media superior de 85\% de estrés académico.

En cuanto a los estresores, el puntaje más alto correspondió a tiempo para cumplir actividades académicas coincidiendo con Polo, Hernández y Poza (7) y con el estudio de Barraza el 2003 (16) que ubica el tiempo limitado para el trabajo como uno de los estresores más altos.

Así podemos observar que no son las evaluaciones las que generan mas estrés, sino la relación cantidad de trabajo/tiempo, es decir los estudiantes perciben que tienen muchas cosas por hacer y poco tiempo para hacerlas.

Respecto a las reacciones físicas nuestros resultados coinciden con Barraza (15) que determinó que la somnolencia es la reacción física con mayor puntaje y los problemas de digestión tienen el menor puntaje en alumnos de educación media superior.

En cuanto a las reacciones psicológicas nuestros resultados coinciden con los estudios de Barraza en 2003 (16) y 2005 (15) quienes encontraron que la incapacidad para relajarse y estar tranquilo fueran las reacciones con mayor puntuación e igualmente la sensación de tener la mente vacía fue la reacción psicológica que presentó menor puntaje. Igualmente se pudo establecer que las reacciones psicológicas como respuesta al estrés se presentan con mayor intensidad en los alumnos, coincidiendo con Polo, Hernández y Poza (7) y Barraza (15)

Queda establecido que el estrés informado por los alumnos es fundamentalmente psicológico, es decir los alumnos "piensan" de forma negativa o se preocupan ante determinadas situaciones académicas.

Es interesante destacar que las situaciones relacionadas a la falta de tiem- po para cumplir las actividades y la sobrecarga académica son las que provocan un mayor nivel de estrés, ello podría confirmar el llamado "Modelo de control", en el sentido de que la sensación de no poder abarcar todo lo que han de hacer, acentua la sensación de falta de control.

El mayor nivel de estrés informado por los alumnos del tercer año parece apoyar esta falta de control en lo referente a la no posesión de probables soluciones a los nuevos requerimientos que tienen que abordar por su comienzo en el manejo de pacientes en las asignaturas.

En relación a las situaciones estresantes parece que los alumnos del cuarto año han desarrollado habilidades que hacen que descienda el nivel de estrés percibido. En este sentido, sería importante constatar que tipo de habilidades y/o estrategias ponen en marcha los alumnos mas "experimentados" para mitigar la sensación de estrés, además de los beneficios que estos puedan acarrear con el paso del tiempo. Dentro de este grupo se encontrarían los alumnos de años superiores, así como aquellos con mas años en la Facultad, que llevan cursos por segunda vez, siendo necesario discriminar la variabilidad de respuestas sobre todo porque en muchos casos, estos alumnos se muestran con menor interés respecto a las obligaciones académicas o por el contrario, otros alumnos pueden denotar mayor preocupación e interés, por ser la aprobación obligatoria de las asignaturas las determinantes de su permanencia en la universidad.

Asimismo, resultaría interesante determinar la presencia de fuentes de estrés no académicas que podrían condicionar y/o predisponer a los alumnos a presentar estrés ante situaciones estresantes en el ámbito académico.

\section{Conclusiones}

- La frecuencia de estrés académico autopercibido por los alumnos es alto.

- Los niveles de estrés fueron mayores en los varones y en los alumnos del tercer año.

- Las situaciones generadoras de estrés ("estresores") atribuyen mayores puntajes al tiempo para cumplir las actividades académicas, sobrecarga de trabajos de cursos y responsabilidad por labores académicas.

\section{Referencias bibliográficas}

1. Ortega ME, Pérez MA. Bases para una psicología del medio. Bucaramanga: Oriente Editores Gráficos; 1996.

2. Looker T, Gregson O. Superar el estrés. Madrid: Pirámide; 1998.

3. Hernández J, Polo A, Poza C. La ansiedad ante los exámenes. Un programa para su tratamiento de forma eficaz. Valencia: Promolibro; 1994.

4. Fisher S. Stress in academic life: the mental assembly line. Bristol: Society for Research into Higher Education and Open University Press; 1994.

5. Kiecolt-Glaser JK, Glaser R, Strain EC, Stout JC, Tarr KL, Holliday JE, Speicher CE. Modulation of cellular immunity in medical students. J Behav Med 1986; 9(1):5-21.

6. Glaser R, Lafuse WP, Bonneau RH, Atkinson C, Kiecolt-Glaser JK. Stress-associated modulation of proto-oncogene expression in human peripheral blood leukocytes. Behav Neurosci 1993; 107(3):525-9.

7. Polo A, Hernández J, Poza C. Evaluación del estrés académico en estudiantes universitarios. Revista Ansiedad y Estrés 1996. Disponible en : http://www.unileon.es/estudiantes/atención_universitario articulo.pdf

8. Fisher S, Hood B. The stress of the transition to university: a longitudinal study of psychological disturbance, absent-mindedness and vulnerability to homesickness. Br J Psychol 1987; 78(Pt 4):425-41.

9. Goldstein MB. Sources of stress and interpersonal support among firstyear dental students. J Dent Educ 1979; 43(12):625-9.

10. Garbee WH Jr, Zucker SB, Selby GR. Perceived sources of stress among dental students. J Am Dent Assoc 1980; 100(6):853-7.

11. George JM, Whitworth DE, Sturdevant JR, Lundeen TF. 
Correlates of dental student stress. J Dent Educ 1987; 51(8):481-5.

12. Martinez, N. Assesment of negative effects in dental students. J Dent Educ 1977; 41 : 31.

13. Wexler M. Mental health and dental education. J Dent Educ 1978; 42(2):74-7.

14. Sturdevant JR, George JM, Lundeen TF. An interactional view of dental student stress. J Dent Educ 1987; 51(5):246-9.
15. Barraza A. Características del estrés académico en los alumnos de educación media superior. Psicología Científica 2005. Disponible en : http://www.ull.es/publicaciones /latina/psicologíacientífica/2004/septiembre.

16. Barraza A. El estrés académico en los alumnos de postgrado de la Universidad Pedagógica de Durango, Guadalajara; 2003. Memoria electrónica del VII Congreso Nacional de
Investigación Educativa; disponible en la Biblioteca Virtual de Psicolnet en : http://www.psicologiacientifica.com/publicaciones/biblioteca/ articulos/arbarraza01.htm.

17. Astudillo C, Avendaño C, Barco M, Franco A, Mosquera C. Efectos biopsicosociales del estrés en estudiantes de la Pontificia Universidad Javeriana de Santiago de Cali; 2003. Disponible en : http//www.puj. edu.co/fhumanidades/psicología/. 